\title{
Chronic Kidney Disease-Mineral Bone Disoder: Fibroblast Growth Factor-23 and Phosphate Metabolism
}

\author{
${ }^{1}$ Wisit Cheungpasitporn, ${ }^{1}$ Pongsathorn Kue-A-Pai, \\ ${ }^{1}$ Patompong Ungprasert, ${ }^{1}$ Wonngarm Kittanamongkolchai, \\ ${ }^{1}$ Narat Srivali, ${ }^{1}$ Supawat Ratanapo, ${ }^{1}$ Teeranun Jirajariyavej, \\ ${ }^{1}$ Daych Chongnarungsin and ${ }^{2}$ Atipon Kangwanpornsiri \\ ${ }^{1}$ Department of Internal Medicine, \\ Bassett Medical Center, Cooperstown, New York, USA \\ ${ }^{2}$ Department of Internal Medicine, Division of Nephrology, \\ University of Rochester Medical Center, New York, USA
}

Received 2012-05-24, Revised 2012-07-10; Accepted 2013-05-04

\begin{abstract}
Chronic Kidney Disease (CKD) is a growing epidemic in the United States. There are hormonal changes that develop long before the mineral changes in patients with CKD occur. Increased Parathyroid Hormone (PTH) levels first become evident when the estimated Glomerular Filtration Rate (eGFR) is below 60 $\mathrm{mL} / \mathrm{min} / 1.73 \mathrm{~m}^{2}$. High serum phosphate stimulates the secretion of the Fibroblast Growth Factor 23 (FGF23) predominantly by bone osteocytes. Recent finding shows that chronically elevated FGF-23 levels in CKD patients are important for the high rates of $\mathrm{LVH}$ and the high rates of mortality. Managing phosphorus disorders with phosphate binders and secondary hyperparathyroidism with vitamin D analog and calcimimetics may theoretically reduce cardiovascular morbidity and mortality. We still need more studies on managing phosphorus disorders with phosphate binders, secondary hyperparathyroidism with vitamin $\mathrm{D}$ analog and calcimimetics and the outcome data on mortality and fractures in CKD patients.
\end{abstract}

Keywords: Chronic Kidney Disease (CKD), Phosphate Metabolism, Fibroblast Growth Factor Receptor 23 (FGF-23)

\section{INTRODUCTION}

Chronic Kidney Disease (CKD) is a growing epidemic in the United States, driven by obesity, hypertension, smoking and lipid disorders. Kidney Disease: Improving Global Outcomes (KDIGO) has defined CKD-MBD as a complex interaction of both laboratory abnormalities, bone disease and vascular disease (KDIGO, 2009).

There are hormonal changes that develop long before the mineral changes in patients with CKD occur. Increased Parathyroid Hormone (PTH) levels first become evident when the estimated Glomerular
Filtration Rate (eGFR) is below $60 \mathrm{~mL} / \mathrm{min} / 1.73 \mathrm{~m}^{2}$ (Levin et al., 2007). Recently, there are also data that fibroblast growth factor-23 (FGF-23) levels rise early in CKD. Plasma calcitriol (1,25-dihydroxyvitamin D levels) also fall early in mild to moderate CKD (GFR 40 to $80 \mathrm{~mL} \mathrm{~min}^{-1}$ ) and is clearly below normal when

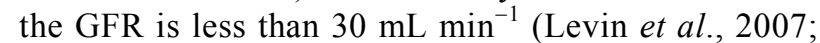
Llach, 1995; Koenig et al., 1992; Wilson et al., 1985; Gutierrez et al., 2008). Those things happen before the changes in calcium and phosphorus. At that time, serum calcium and phosphate levels remain within normal ranges until eGFR decrease to approximately 20 $\mathrm{mL} / \mathrm{min} / 1.73 \mathrm{~m}^{2}$ (Levin et al., 2007). 


\subsection{Fibroblast Growth Factor-23 (FGF 23)}

Fibroblast Growth Factor 23 (FGF-23) is a circulating peptide that regulates phosphate (Cunningham et al., 2011; Liu et al., 2007; Liu and Quarles, 2007). High serum phosphate stimulates the secretion of the FGF-23 predominantly by bone osteocytes which then act on its target tissues by binding to and activating its receptor. Klotho, a transmembrane protein produced by the osteocyte, is required for FGF-23 receptor activation (Urakawa et al., 2006). The klotho extracellular domain enhances FGF23 binding to its receptor complex with a much higher affinity than to Fibroblast Growth Factor Receptor (FGFR) alone (Kuro-o, 2006).

Klotho is primarily expressed in the distal convoluted tubules where it exerts some signaling effects (Farrow et al., 2009); however, its major action is on proximal tubule cells (John et al., 2011). PTH and Klotho increase FGF-23 production by osteocytes, while FGF-23 inhibits PTH secretion, thus forming an important activation pathway with negative feedback control (Krajisnik et al., 2010). Actions of Klotho include activation of transient receptor potential calcium channels (TRPVs), especially TRPV5 and TRPV6 (Cha et al., 2008). TRPV5 is expressed in distal nephrons and mediates calcium reabsorption at that site. TRPV6 is expressed in small intestinal epithelial cells where it mediates calcium absorption.

There are two main actions of FGF-23 on the kidney. Firstly, it allows the kidney to excrete more phosphate and brings the phosphate back down. It suppresses the $\mathrm{Na} / \mathrm{Pi}$ co-transporter activity in the renal proximal tubular cells and subsequently reduces renal phosphate reabsorption and increases urinary phosphate excretion (Miyamoto et al., 2007). Secondly, FGF-23 regulates calcitriol production, which regulates phosphate absorption. It inhibits the 1-alpha-hydroxylase enzyme and leads to decreased calcitriol synthesis by the kidney (Saito et al., 2003). Patients with CKD have significantly elevated FGF-23 levels due to phosphate retention and decreased clearance (Gutierrez et al., 2005; Imanishi et al., 2004; Larsson et al., 2003).

The parathyroid gland is another target organ for FGF-23, which acts on the parathyroid gland to suppress PTH secretion (Ben-Dov et al., 2007). However, the presence of high PTH levels in CKD despite high FGF-23 levels suggests that the parathyroid is relatively resistant to the elevated levels of FGF-23 in uremia.
In the past, cardiorenal syndromes were focused mainly on how cardiac pump function was altered by kidney changes. Now we are learning that, in patients with CKD, elevated FGF-23 is independently associated with left Ventricular Hypertrophy (LVH) (Faul et al., 2011; Stevens et al., 2011). This finding shows that chronically elevated FGF-23 levels in these patients are important for the high rates of $\mathrm{LVH}$ and the high rates of mortality that we see in individuals with CKD. Currently, maintaining normal levels of phosphate with phosphate binders is the main treatment for FGF-23 elevation.

\subsection{Phosphate Homeostasis and Alterations in CKD}

In the steady state, the serum phosphate concentration is primarily determined by the ability of the kidneys to excrete dietary phosphate. Renal excretion is so efficient in normal subjects that balance can be maintained with only a minimal rise in serum phosphate concentration even if phosphate intake is increased to as much as $4000 \mathrm{mg} \mathrm{day}^{-1}$ (130 $\mathrm{mmol} /$ day). This response is in part mediated by a direct effect of minimal hyperphosphatemia to diminish proximal tubular phosphate reabsorption, via inhibition of sodium-phosphate cotransporters in the luminal membrane that allow reabsorption of filtered phosphate (Murer, 1992; Murer et al., 1996).

Increased secretion of Parathyroid Hormone (PTH) also may contribute to the reduction in phosphate reabsorption, because some of the excess phosphate may complex with calcium in the serum. The ensuing fall in serum ionized calcium concentration provides the signal for increased PTH release.

\subsection{Options for Managing Phosphorus Disorders: Phosphate Binders}

The 2 main categories of phosphate binders (Table 1) are calcium-based and non-calcium-based. The commonly used calcium-based ones are calcium acetate, which is $25 \%$ elemental calcium and calcium carbonate, which is $40 \%$ elemental calcium. With calcium-based binders, there is always the possibility of hypercalcemia or perhaps progressing calcium phosphate deposition if that is present. The noncalcium-based binders are sevelamer, in the forms of sevelamer hydrochloride and sevelamer carbonate and lanthanum carbonate. These bind phosphate as anion exchangers and do not necessarily have the effect of hypercalcemia in patients. 
Table 1. Phosphate binders

\begin{tabular}{ll}
\hline Calcium based & Calcium free \\
\hline -Calcium acetate $(25 \%$ & -Sevelamer hydrochloride \\
elemental calcium) & -Sevelamer carbonate \\
-Calcium carbonate $(40 \%$ & -Lanthanum carbonate \\
elemental calcium) & \\
\hline
\end{tabular}

However, there are limited outcome data to suggest use of one phosphate binder over another. Studies comparing calcium-based and non-calcium-based binders have investigated mortality, vascular calcification and bone outcomes. While some of these studies have shown a benefit of using the non-calciumbased binders such as sevelamer over calcium carbonate, others have shown no difference. There is still uncertainty about what the data are showing us, so we need more studies with hard outcome data on mortality and fractures to be able to determine which phosphate binders are best to use. Also, patients with PTH or serum phosphate levels greater than target levels should be on dietary phosphate restriction to $900 \mathrm{mg} \mathrm{day}^{-1}$.

\subsection{Management of Hyperparathyroidism}

In the past, the management has been to do a surgical parathyroidectomy, or to do a partial procedure so that you leave some of the parathyroid tissue in and bring down the parathyroid blood level. Currently, there are 2 classes of drugs that allow us to attempt a medical parathyroidectomy.

\subsection{Drugs Based on 1,25-Dihydroxyvitamin D}

In addition to increasing calcium absorption, these drugs act to decrease the secretion of PTH directly. A number of these analogues drugs (calcitriol, alfacalcidol, doxercalciferol, or paricalcitol) have been developed. The goal in using these is to bring down the serum PTH and not to increase calcium absorption from the gut or calcium resorption and phosphate resorption from bone. These agents are very effective. However, treatment with a vitamin $\mathrm{D}$ analog should not be given to predialysis patients with stage 3-5 CKD unless the serum phosphate is in the normal range and the corrected serum total calcium concentration is less than $9.5 \mathrm{mg} \mathrm{dL}^{-1}$.

If the serum level of corrected total calcium exceeds $10.2 \mathrm{mg} \mathrm{dL}^{-1}$, ergocalciferol therapy and all forms of vitamin D therapy should be discontinued. Vitamin D therapy should also be discontinued if intact PTH levels become persistently low.

\subsection{Calcimimetics}

Calcimimetics are agents act on the CalciumSensing Receptor (CaSR), which is present in a number of tissues, including the parathyroid gland. The allosteric calcimimetics sensitize the gland to the presence of calcium (Nemeth and Bennett, 1998) and so they decrease PTH. There are 2 different mechanisms involved. One acts on PTH directly and also increases calcium and phosphate absorption from gut, whereas the other one decreases PTH but does so in a pulsatile fashion. Where there is kidney function present, this has an effect on increasing bone turnover markers. So, they have a slightly different action, as one might think.

Cinacalcet (Sensipar), the only current available calcimimetic, could be useful in predialysis patients with secondary hyperparathyroidism that is refractory to therapy with vitamin D analogues, calcium supplements and phosphate binders. Cinacalcet used with lower doses of active vitamin D reduces FGF23 concentrations, unlike active vitamin D analogues alone, which can increase circulating FGF23 concentrations (Wetmore et al., 2010). Cinacalcet is indicated in all dialysis patients with PTH levels $>300$ $\mathrm{pg} / \mathrm{mL}$ who have serum calcium levels $>8.4 \mathrm{mg} \mathrm{dL}^{-1}$

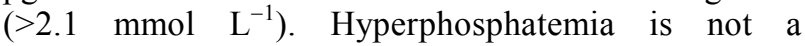
contraindication for starting cinacalcet, unlike vitamin $\mathrm{D}$ analogues. However, hypocalcemia and elevations of serum phosphate are observed in the use of cinacalcet and requiring for close monitoring.

\section{CONCLUSION}

Deranged phosphate metabolism in CKD is associated with the increased cardiovascular morbidity and mortality as well as progression of renal insufficiency. Serum PTH and FGF-23 increase in response to rising serum phosphate as renal capacity for phosphate excretion is lost. Managing phosphorus disorders with phosphate binders and secondary hyperparathyroidism with vitamin $\mathrm{D}$ analog and calcimimetics may theoretically reduce cardiovascular morbidity and mortality. We still need more studies and outcome data of these agents on mortality in CKD patients.

\subsection{Disclosure Statement}

The authors have nothing to disclose.

\section{ACKNOWLEDGEMENT}

We acknowledge Dr.Edward Bischof, Program Director of the Internal Medicine Residency Program 
and Dr.Eric Knight, Nephrology attending at Bassett Medical Center, who always encourage us to apply evidence-based medicine to clinical practice.

\section{REFERENCES}

Ben-Dov, I.Z., H. Galitzer, V. Lavi-Moshayoff, R. Goetz and M. Kuro-o et al., 2007. The parathyroid is a target organ for FGF23 in rats. J. Clin. Invest., 117: 4003-4008. PMID: 17992255

Cha, S.K., B. Ortega, H. Kurosu, K.P. Rosenblatt and M. Kuro-o et al., 2008. Removal of sialic acid involving Klotho causes cell-surface retention of TRPV5 channel via binding to galectin-1. Proc. Natl. Acad. Sci. USA., 105: 9805-9810. PMID: 18606998

Cunningham, J., F. Locatelli and M. Rodriguez, 2011. Secondary hyperparathyroidism: Pathogenesis, disease progression and therapeutic options. Clin. J. Am. Soc. Nephrol., 6: 913-921. PMID: 21454719

Farrow, E.G., S.I. Davis, L.J. Summers and K.E. White, 2009. Initial FGF23-mediated signaling occurs in the distal convoluted tubule. J. Am. Soc. Nephrol., 20: 955-960. PMID: 19357251

Faul, C., A.P. Amaral, B. Oskouei, M.C. Hu and A. Sloan et al., 2011. FGF23 induces left ventricular hypertrophy. J. Clin. Invest., 121: 4393-4408. DOI: 10.1172/JCI46122

Gutierrez, O., T. Isakova, E. Rhee, A. Shah and J. Holmes, et al., 2005. Fibroblast growth factor-23 mitigates hyperphosphatemia but accentuates calcitriol deficiency in chronic kidney disease. J. Am. Soc. Nephrol., 16: 2205-2215. PMID: 15917335

Gutierrez, O.M., T. Isakova D.L. Andress, A. Levin and M. Wolf, 2008. Prevalence and severity of disordered mineral metabolism in Blacks with chronic kidney disease. Kidney Int., 73: 956-962. PMID: 18256597

Imanishi, Y., M. Inaba, K. Nakatsuka, K. Nagasue and S. Okuno et al., 2004. FGF-23 in patients with endstage renal disease on hemodialysis. Kidney Int., 65: 1943-1946. PMID: 15086938

John, G.B., C.Y. Cheng and M. Kuro-o, 2011. Role of Klotho in aging, phosphate metabolism and CKD. Am. J. Kidney Dis., 58: 127-134. PMID: 21496980

KDIGO, 2009. KDIGO clinical practice guideline for the diagnosis, evaluation, prevention and treatment of chronic kidney disease-mineral and bone disorder (CKD-MBD). Kidney Int. Suppl., 113: S1-S130. PMID: 19644521
Koenig, K.G., J.S. Lindberg, J.E. Zerwekh, P.K. Padalino and H.M. Cushner et al., 1992. Free and total 1,25-dihydroxyvitamin D levels in subjects with renal disease. Kidney Int., 41: 161-165. PMID: 1593853

Krajisnik, T., H. Olauson, M.A. Mirza, P. Hellman and G. Akerstrom, et al., 2010. Parathyroid klotho and FGF-receptor 1 expression decline with renal function in hyperparathyroid patients with chronic kidney disease and kidney transplant recipients. Kidney Int., 78: 1024-1032. PMID: 20686451

Kuro-o, M., 2006. Klotho as a regulator of fibroblast growth factor signaling and phosphate/calcium metabolism. Curr. Opin. Nephrol. Hypertens, 15: 437-441. PMID: 16775459

Larsson, T., U. Nisbeth, O. Ljunggren, H. Juppner and K.B. Jonsson, 2003. Circulating concentration of FGF-23 increases as renal function declines in patients with chronic kidney disease, but does not change in response to variation in phosphate intake in healthy volunteers. Kidney Int., 64: 2272-2279. PMID: 14633152

Levin, A., G.L. Bakris, M. Molitch, M. Smulders and J. Tian et al., 2007. Prevalence of abnormal serum vitamin D, PTH, calcium and phosphorus in patients with chronic kidney disease: Results of the study to evaluate early kidney disease. Kidney Int., 71: 3131. PMID: 17091124

Liu, S. and L.D. Quarles, 2007. How fibroblast growth factor 23 works. J. Am. Soc. Nephrol., 18: 16371647. PMID: 17494882

Liu, S., A. Gupta and L.D. Quarles, 2007. Emerging role of fibroblast growth factor 23 in a bone-kidney axis regulating systemic phosphate homeostasis and extracellular matrix mineralization. Curr. Opin. Nephrol. Hypertens, 16: 329-335. PMID: 17565275

Llach, F., 1995. Secondary hyperparathyroidism in renal failure: The trade-off hypothesis revisited. Am. J. Kidney Dis., 25: 663-679. PMID: 7747720

Miyamoto, K., M. Ito, S. Tatsumi, M. Kuwahata and H. Segawa, 2007. New aspect of renal phosphate reabsorption: The type IIc sodium-dependent phosphate transporter. Am. J. Nephrol., 27: 503-515. PMID: 17687185

Murer, H., 1992. Homer Smith Award. Cellular mechanisms in proximal tubular $\mathrm{Pi}$ reabsorption: Some answers and more questions. J. Am. Soc. Nephrol., 2: 1649-1665. PMID: 1498272 
Murer, H., M. Lotscher, B. Kaissling, M. Levi and S.A. Kempson et al., 1996. Renal brush border membrane $\mathrm{Na} / \mathrm{Pi}$-cotransport: molecular aspects in PTH-dependent and dietary regulation. Kidney Int., 49:1769-1773. PMID: 8743494

Nemeth, E.F. and S.A. Bennett, 1998. Tricking the parathyroid gland with novel calcimimetic agents. Nephrol. Dial. Transplant, 13: 1923-1925. PMID: 9719139

Saito, H., K. Kusano, M. Kinosaki, H. Ito and M. Hirata et al., 2003. Human fibroblast growth factor-23 mutants suppress $\mathrm{Na}+$-dependent phosphate cotransport activity and 1alpha, 25-dihydroxyvitamin D3 production. J. Biol. Chem., 278: 2206-2211. PMID: 12419819

Stevens, K.K., E.P. McQuarrie, W. Sands, D.Z. Hillyard and R.K. Patel et al., 2011. Fibroblast growth factor 23 predicts left ventricular mass and induces cell adhesion molecule formation. Int. J. Nephrol., 2011: 297070-297070. DOI: 10.4061/2011/297070
Urakawa, I., Y. Yamazaki, T. Shimada, K. Iijima and H. Hasegawa et al., 2006. Klotho converts canonical FGF receptor into a specific receptor for FGF23. Nature, 444: 770-774. DOI: 10.1038/nature05315

Wetmore, J.B., S. Liu, R. Krebill, R. Menard and L.D. Quarles, 2010. Effects of cinacalcet and concurrent low-dose vitamin D on FGF23 levels in ESRD. Clin. J. Am. Soc. Nephrol., 5:110-116. PMID: 19965548

Wilson, L., A. Felsenfeld, M.K. Drezner and F. Llach, 1985. Altered divalent ion metabolism in early renal failure: Role of 1,25(OH)2D. Kidney Int., 27: 565573. PNID: 3839029 